\title{
Partial Ordering of Range Symmetric Matrices and M-Projectors with Respect to Minkowski Adjoint in Minkowski Space
}

\author{
D. Krishnaswamy, Mohd Saleem Lone \\ Department of Mathematics, Annamalai University, Chidambaram, India \\ Email: krishna_swamy2004@yahoo.co.in, saleemlone9@gmail.com
}

How to cite this paper: Krishnaswamy, D. and Lone, M.S. (2016) Partial Ordering of Range Symmetric Matrices and M-Projectors with Respect to Minkowski Adjoint in Minkowski Space. Advances in Linear Algebra \& Matrix Theory, 6, 132-145.

http://dx.doi.org/10.4236/alamt.2016.64013

Received: October 6, 2016

Accepted: December 3, 2016

Published: December 6, 2016

Copyright $\odot 2016$ by authors and Scientific Research Publishing Inc. This work is licensed under the Creative Commons Attribution International License (CC BY 4.0).

http://creativecommons.org/licenses/by/4.0/

\begin{abstract}
In this paper, we obtain some new characterizations of the range symmetric matrices in the Minkowski Space $\mathcal{M}$ by using the Block representation of the matrices. These characterizations are used to establish some results on the partial ordering of the range symmetric matrices with respect to the Minkowski adjoint. Further, we establish some results regarding the partial ordering of m-projectors with respect to the Minkowski adjoint and manipulate them to characterize some sets of range symmetric elements in the Minkowski Space $\mathcal{M}$. All the results obtained in this paper are an extension to the Minkowski space of those given by A. Hernandez, et al. in [The star partial order and the eigenprojection at 0 on EP matrices, Applied Mathematics and Computation, 218: 10669-10678, 2012].
\end{abstract}

\section{Keywords}

Partial Order, Minkowski Adjoint, Minkowski Inverse, Range Symmetric, M-Projectors

\section{Introduction and Preliminaries}

Let us denote by $M_{(m, n)}(\mathbb{C})$ the set of $m \times n$ matrices and when $m=n$ we write $M_{n}(\mathbb{C})$ for $M_{(n, n)}(\mathbb{C})$. The symbols $\boldsymbol{A}^{*}, \boldsymbol{A}^{\sim}, \boldsymbol{A}^{\oplus}, \boldsymbol{A}^{\dagger}, R(\boldsymbol{A})$ and $N(\boldsymbol{A})$ denote the conjugate transpose, Minkowski adjoint, Minkowski inverse, Moore-Penrose inverse, range space and null space of a matrix $\boldsymbol{A}$ respectively. $\boldsymbol{I}_{n}$ denote the identity matrix of order $n \times n$. Further we denote by $\mathbb{C}_{n}^{m p}$ the set of all $m$-projections. i.e. $\mathbb{C}_{n}^{m p}=\left\{\boldsymbol{P}: \boldsymbol{P}^{2}=\boldsymbol{P}=\boldsymbol{P}^{\sim}\right\}$. Also we use the convection according to which $\boldsymbol{P}_{w}=\boldsymbol{W} \boldsymbol{W}^{\oplus}$ and $\tilde{\boldsymbol{P}}_{w}=\boldsymbol{I}_{k}-\boldsymbol{W} \boldsymbol{W}^{\oplus}$. Where $\boldsymbol{I}_{k}$ is the identity matrix of suitable order. $r$ and $s$ will denote the rank of the matrices $\boldsymbol{R}$ and $S$. 
Indefinite inner product is a scalar product defined by

$$
[u, v]=\langle u, M v\rangle=u^{*} M v
$$

where $\langle$,$\rangle denotes the conventional Hilbert Space inner product and \boldsymbol{M}$ is a Hermitian matrix. This Hermitian matrix $\boldsymbol{M}$ is referred to as metric matrix. Minkowski Space $\mathcal{M}$ is an indefinite inner product space in which the metric matrix is denoted by $\boldsymbol{G}$ and is defined as $\boldsymbol{G}=\left[\begin{array}{cc}1 & 0 \\ 0 & -\boldsymbol{I}_{n-1}\end{array}\right]$ satisfying $\boldsymbol{G}^{2}=\boldsymbol{I}_{n}$ and $\boldsymbol{G}^{*}=\boldsymbol{G}$.

$\boldsymbol{G}$ is called the Minkowski metric matrix. In case $\boldsymbol{u}=\left(u_{0}, u_{1}, \cdots, u_{n-1}\right) \in \mathbb{C}^{n}$, then $\boldsymbol{G}$ is called the Minkowski metric tensor and is defined as $\boldsymbol{G} \boldsymbol{u}=\left(u_{0},-u_{1}, \cdots,-u_{n-1}\right)$. For detailed study of indefinite linear algebra refer to [1].

The minkowski inverse of a matrix $\boldsymbol{R} \in M_{(m, n)}(\mathbb{C})$, introduced by Meenakshi in [2], is the unique solution to the following four matrix equations:

[MI-1]: $\quad \boldsymbol{R X} \boldsymbol{R}=\boldsymbol{R}$.

[MI-2]: $\quad X R X=X$.

[MI-3]: $(\boldsymbol{R X})^{\sim}=\boldsymbol{R} \boldsymbol{X}$.

[MI-4]: $(X \boldsymbol{R})^{\sim}=X \boldsymbol{R}$.

However unlike the Moore-Penrose inverse of a matrix, the Minkowski inverse of a matrix does not exist always. In [2], Meenakshi showed that the Minkowski inverse of a matrix $\boldsymbol{R} \in M_{(m, n)}(\mathbb{C})$ exists if and only if $\operatorname{rk}\left(\boldsymbol{R} \boldsymbol{R}^{\sim}\right)=\operatorname{rk}\left(\boldsymbol{R}^{\sim} \boldsymbol{R}\right)=\operatorname{rk}(\boldsymbol{R})$, where $\boldsymbol{R}^{\sim}=\boldsymbol{G}_{1} \boldsymbol{R}^{*} \boldsymbol{G}_{2}$ is called the Minkowski adjoint of the matrix $\boldsymbol{R}$ and $\boldsymbol{G}_{1}$ and $\boldsymbol{G}_{2}$ are the Minkowski metric matrices of suitable order $m$ and $n$. A matrix $\boldsymbol{R} \in M_{n}(\mathbb{C})$ is said to be m-symmetric if $\boldsymbol{R}=\boldsymbol{R}^{\sim}$ and is said to be G-unitary if and only if

$\boldsymbol{R} \boldsymbol{R}^{\sim}=\boldsymbol{R}^{\sim} \boldsymbol{R}=\boldsymbol{I}_{n}$. In [3], Meenakshi introduced the concept of range symmetric matrices in Minkowski Space and developed the Minkowski inverse of the range symmetric matrices and some equivalent conditions for a matrix to be range symmetric. A matrix $R \in M_{n}(\mathbb{C})$ is said to be range symmetric if and only if $N(\boldsymbol{R})=N\left(\boldsymbol{R}^{\sim}\right)$. In [4], the authors produced the necessary and sufficient conditions for the product of range symmetric matrices to be range symmetric and further showed that any block matrix in Minkowski space can be expressed as the product of range symmetric matrices. In [5] the authors studied the range symmetric matrices in relation with their Minkowski inverse and m-projectors. Summarizing the equivalent conditions for the definition of a range symmetric matrix form [3] [5] [6] the following equivalent conditions will be used in the forthcoming results:

[RS-1]: $\boldsymbol{R}$ is range symmetric.

[RS-2]: $N(\boldsymbol{R})=N\left(\boldsymbol{R}^{\sim}\right)$.

[RS-3]: $\quad \boldsymbol{R} \boldsymbol{R}^{\oplus}=\boldsymbol{R}^{\oplus} \boldsymbol{R}$.

[RS-4]: $R(\boldsymbol{R})=R\left(\boldsymbol{R}^{\sim}\right)$.

[RS-5]: their exist a G-unitary matrix $\boldsymbol{U}$ such that $\boldsymbol{R}=\boldsymbol{U}_{R}\left(\boldsymbol{D}_{R} \oplus 0\right) \boldsymbol{U}_{R}^{\sim}$.

Partial orders on matrices has remained the topic of interest for many authors in the area of matrix theory and generalized inverse. Almost all authors who have worked on partial ordering of matrices have formulated the definition involving different kinds of 
generalized inverses and in particular the Moore-Penrose Inverse. Results involving partial orders on matrices in relation with their generalized inverse are scattered in the literature of the matrix theory and generalized inverses for instance see [7]-[19]. Partial ordering on matrices has a wide range of applications in different fields which include electrical networks, statistics, generalized inverses etc. see [20] [21] [22] [23]. Different kinds of partial orders on matrices have been studied which include Star partial ordering $\leq^{*}$ introduced by Drazin [24], minus partial order $\leq^{-}$introduced by Hartwig [25], Sharp partial $\leq^{\#}$ order introduced by Mitra [19], followed by left star ordering * $\leq$ and right star ordering $\leq *$. In [26], Punithavalli introduced the partial ordering on matrices in Minkowski space w.r.t the Minkowski adjoint. She studied the partial ordering, left partial ordering and right partial ordering w.r.t the Minkowski adjoint on Range symmetric matrices. She also established some equivalent conditions for the reverse order law to hold in relation to the partial ordering w.r.t Minkowski adjoint. Form ([26], page 79), we have for any two matrices $\boldsymbol{R}, \boldsymbol{S} \in M_{(m, n)}(\mathbb{C}), \boldsymbol{R}$ is said to be below $\boldsymbol{S}$ under the partial order w.r.t Minkowski adjoint, denoted by $\boldsymbol{R} \leq^{\sim} \boldsymbol{S}$, if one of the following equivalent condition is satisfied:

[PO-1]: $\quad \boldsymbol{R}^{\sim} \boldsymbol{R}=\boldsymbol{R}^{\sim} \boldsymbol{S}$ and $\boldsymbol{R} \boldsymbol{R}^{\sim}=\boldsymbol{S} \boldsymbol{R}^{\sim}$.

[PO-2]: $\quad \boldsymbol{R}^{\oplus} \boldsymbol{R}=\boldsymbol{R}^{\oplus} \boldsymbol{S}$ and $\boldsymbol{R} \boldsymbol{R}^{\oplus}=\boldsymbol{S} \boldsymbol{R}^{\oplus}$.

[PO-3]: $R(\boldsymbol{R}) \subset R(\boldsymbol{S})$ and $R\left(\boldsymbol{R}^{\sim}\right) \subset R\left(\boldsymbol{S}^{\sim}\right)$.

In any of the above cases we say $\boldsymbol{R}$ is predecessor of $\boldsymbol{S}$ or $\boldsymbol{S}$ is successor of $\boldsymbol{R}$. We will use the notation $M_{n}^{k}(\mathbb{C})=M_{n}^{k}$ to denote the set of all the matrices of index $k$.

In this paper we obtain some characterizations of range symmetric matrices and utilize them to study the partial ordering of range symmetric matrices w.r.t the Minkowski adjoint in Minkowski space and hence different characterizations of partial orders on range symmetric matrices are obtained. Finally we study the partial ordering on m-Projectors w.r.t the Minkowski adjoint. All the results obtained in this paper are an extension of those given in [27] to the Minkowski space $\mathcal{M}$.

\section{Properties of Range Symmetric Matrices}

In this section we develop some properties of Range Symmetric matrices by utilizing the representation obtained in corollary 2.6 in [5]. Let $\boldsymbol{R}, \boldsymbol{S} \in M_{n}(\mathbb{C})$ be non-zero range symmetric matrices of rank $\boldsymbol{r}$ and $\boldsymbol{s}$ respectively. Then $\boldsymbol{R}$ and $\boldsymbol{S}$, according to the above mentioned result, can be written as

$$
\boldsymbol{R}=\boldsymbol{U}_{R}\left[\begin{array}{cc}
\boldsymbol{D}_{R} & 0 \\
0 & 0
\end{array}\right] \boldsymbol{U}_{R}^{\sim}
$$

and

$$
\boldsymbol{S}=\boldsymbol{U}_{S}\left[\begin{array}{cc}
\boldsymbol{D}_{S} & 0 \\
0 & 0
\end{array}\right] \boldsymbol{U}_{S}^{\sim}
$$

where $\boldsymbol{U}_{R}$ and $\boldsymbol{U}_{S}$ are G-unitary and $\boldsymbol{D}_{R}$ and $\boldsymbol{D}_{S}$ are invertible matrices of order $r$.

Theorem 1 Let $\boldsymbol{R}, \boldsymbol{S} \in M_{n}(\mathbb{C})$ be such that $\boldsymbol{R}$ is range symmetric. Then the fol- 
lowing statements are equivalent.

1. $\boldsymbol{R S}=\boldsymbol{S R}$

2. If $\boldsymbol{R}$ is given by (2), then there exists $\boldsymbol{J} \in M_{r}(\mathbb{C})$ and $\boldsymbol{M} \in M_{n-r}(\mathbb{C})$ such that $\boldsymbol{S}=\boldsymbol{U}_{R}\left[\begin{array}{cc}\boldsymbol{J} & 0 \\ 0 & \boldsymbol{M}\end{array}\right] \boldsymbol{U}_{R}^{\sim}$ with $\boldsymbol{D}_{R} \boldsymbol{J}=\boldsymbol{J D}_{R}$

Proof. We consider the decomposition of the matrix $S$, according to the size of blocks of $\boldsymbol{R}$, as:

$$
\boldsymbol{S}=\boldsymbol{U}_{R}\left[\begin{array}{ll}
\boldsymbol{J} & \boldsymbol{K} \\
\boldsymbol{L} & \boldsymbol{M}
\end{array}\right] \boldsymbol{U}_{R}^{\sim} .
$$

From the statement (i) of the theorem, we get

$$
\left[\begin{array}{cc}
\boldsymbol{D}_{R} & 0 \\
0 & 0
\end{array}\right]\left[\begin{array}{cc}
\boldsymbol{J} & \boldsymbol{K} \\
\boldsymbol{L} & \boldsymbol{M}
\end{array}\right]=\left[\begin{array}{cc}
\boldsymbol{J} & \boldsymbol{K} \\
\boldsymbol{L} & \boldsymbol{M}
\end{array}\right]\left[\begin{array}{cc}
\boldsymbol{D}_{R} & 0 \\
0 & 0
\end{array}\right] .
$$

This gives $\boldsymbol{D}_{R} \boldsymbol{J}=\boldsymbol{J} \boldsymbol{D}_{R}, \quad \boldsymbol{L}=0$ and $\boldsymbol{K}=0$ and hence the result follows.

If both the matrices $\boldsymbol{R}$ and $S$ are range symmetric, then we have the following result for the commutativity.

Theorem 2 Let $\boldsymbol{R}, \boldsymbol{S} \in M_{n}(\mathbb{C})$ be range symmetric matrices. If $\boldsymbol{U}_{R}^{\sim} \boldsymbol{U}_{S}=\left[\begin{array}{ll}\boldsymbol{J} & \boldsymbol{K} \\ \boldsymbol{L} & \boldsymbol{M}\end{array}\right]$ Then the following statements are equivalent.

1. $\boldsymbol{R S}=\boldsymbol{S R}$.

2. $\left(\boldsymbol{J}^{\sim} \boldsymbol{D}_{R} \boldsymbol{J}\right) \boldsymbol{D}_{S}=\left(\boldsymbol{D}_{S} \boldsymbol{J}^{\sim} \boldsymbol{D}_{R}\right) \boldsymbol{J}$.

3. $\boldsymbol{D}_{R}\left(\boldsymbol{J} \boldsymbol{D}_{S} \boldsymbol{J}^{\sim}\right)=\left(\boldsymbol{J} \boldsymbol{D}_{S} \boldsymbol{J}^{\sim}\right) \boldsymbol{D}_{R}$.

Proof. (i) $\Leftrightarrow$ (ii) Consider the representations of $\boldsymbol{R}$ and $S$ given by (2) and (3) respectively. With given $\boldsymbol{U}_{R}^{\sim} \boldsymbol{U}_{S}=\left[\begin{array}{ll}\boldsymbol{J} & \boldsymbol{K} \\ \boldsymbol{L} & \boldsymbol{M}\end{array}\right]$, we have

$$
\boldsymbol{R} \boldsymbol{S}=\boldsymbol{U}_{R}\left[\begin{array}{cc}
\boldsymbol{D}_{R} & 0 \\
0 & 0
\end{array}\right]\left[\begin{array}{cc}
\boldsymbol{J} & \boldsymbol{K} \\
\boldsymbol{L} & \boldsymbol{M}
\end{array}\right]\left[\begin{array}{cc}
\boldsymbol{D}_{S} & 0 \\
0 & 0
\end{array}\right] \boldsymbol{U}_{S}^{\sim}=\boldsymbol{U}_{R}\left[\begin{array}{cc}
\boldsymbol{D}_{R} \boldsymbol{J} \boldsymbol{D}_{S} & 0 \\
0 & 0
\end{array}\right] \boldsymbol{U}_{S}^{\sim}
$$

Also

$$
\boldsymbol{U}_{S}^{\sim} \boldsymbol{U}_{R}=\left(\boldsymbol{U}_{R}^{\sim} \boldsymbol{U}_{S}\right)^{\sim}=\left[\begin{array}{cc}
\boldsymbol{J}^{\sim} & -\boldsymbol{L}^{\sim} \boldsymbol{G}_{r} \\
-\boldsymbol{G}_{r} \boldsymbol{K}^{\sim} & \boldsymbol{G}_{n-r} \boldsymbol{M}^{\sim} \boldsymbol{G}_{n-r}
\end{array}\right]
$$

Therefore

$$
\begin{aligned}
\boldsymbol{S} \boldsymbol{R} & =\boldsymbol{U}_{S}\left[\begin{array}{cc}
\boldsymbol{D}_{S} & 0 \\
0 & 0
\end{array}\right]\left[\begin{array}{cc}
\boldsymbol{J}^{\sim} & -\boldsymbol{L}^{\sim} \boldsymbol{G}_{r} \\
-\boldsymbol{G}_{r} \boldsymbol{K}^{\sim} & \boldsymbol{G}_{n-r} \boldsymbol{M}^{\sim} \boldsymbol{G}_{n-r}
\end{array}\right]\left[\begin{array}{cc}
\boldsymbol{D}_{R} & 0 \\
0 & 0
\end{array}\right] \boldsymbol{U}_{R}^{\sim} \\
& =\boldsymbol{U}_{S}\left[\begin{array}{cc}
\boldsymbol{D}_{S} \boldsymbol{J}^{\sim} \boldsymbol{D}_{R} & 0 \\
0 & 0
\end{array}\right] \boldsymbol{U}_{R}^{\sim}
\end{aligned}
$$

From Equations (4) and (5) we have

$$
\boldsymbol{U}_{R}\left[\begin{array}{cc}
\boldsymbol{D}_{R} \boldsymbol{J} \boldsymbol{D}_{S} & 0 \\
0 & 0
\end{array}\right] \boldsymbol{U}_{S}^{\sim}=\boldsymbol{U}_{S}\left[\begin{array}{cc}
\boldsymbol{D}_{S} \boldsymbol{J}^{\sim} \boldsymbol{D}_{R} & 0 \\
0 & 0
\end{array}\right] \boldsymbol{U}_{R}^{\sim}
$$

Pre multiplying and post multiplying (6) by $\boldsymbol{U}_{S}^{\sim}$ and $\boldsymbol{U}_{S}$ respectively and substituting the matrix representation of $\boldsymbol{U}_{S}^{\sim} \boldsymbol{U}_{R}$ and $\boldsymbol{U}_{R}^{\sim} \boldsymbol{U}_{S}$ we get 


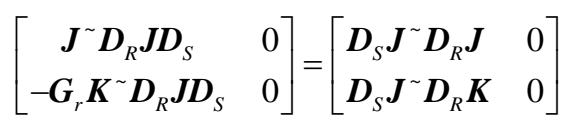

From this equality, on using the fact that $\boldsymbol{D}_{R}$ and $\boldsymbol{D}_{S}$ are nonsingular, we have $\left(\boldsymbol{J}^{\sim} \boldsymbol{D}_{R} \boldsymbol{J}\right) \boldsymbol{D}_{S}=\boldsymbol{D}_{S}\left(\boldsymbol{J}^{\sim} \boldsymbol{D}_{R} \boldsymbol{J}\right), \quad \boldsymbol{K}^{\sim} \boldsymbol{D}_{R} \boldsymbol{J}=0$, and $\boldsymbol{J}^{\sim} \boldsymbol{D}_{R} \boldsymbol{K}=0$ and hence the equivalence follows.

(i) $\Leftrightarrow$ (iii) From $\boldsymbol{U}_{R}^{\sim} \boldsymbol{U}_{S}=\left[\begin{array}{ll}\boldsymbol{J} & \boldsymbol{K} \\ \boldsymbol{L} & \boldsymbol{M}\end{array}\right]$, using the fact that $\boldsymbol{U}_{R}$ is G-unitary, we have $\boldsymbol{U}_{S}=\boldsymbol{U}_{R}\left[\begin{array}{ll}\boldsymbol{J} & \boldsymbol{K} \\ \boldsymbol{L} & \boldsymbol{M}\end{array}\right]$ and hence $\boldsymbol{U}_{S}^{\sim}=\left[\begin{array}{cc}\boldsymbol{J}^{\sim} & -\boldsymbol{L}^{\sim} \boldsymbol{G}_{r} \\ -\boldsymbol{G}_{r} \boldsymbol{K}^{\sim} & \boldsymbol{G}_{n-r} \boldsymbol{M}^{\sim} \boldsymbol{G}_{n-r}\end{array}\right] \boldsymbol{U}_{R}^{\sim}$. Substituting the representations of $\boldsymbol{U}_{S}$ and $\boldsymbol{U}_{S}^{\sim}$ in the block representation of $\boldsymbol{S}$ given by (3) we have

$$
\begin{aligned}
\boldsymbol{S} & =\boldsymbol{U}_{R}\left[\begin{array}{cc}
\boldsymbol{J} & \boldsymbol{K} \\
\boldsymbol{L} & \boldsymbol{M}
\end{array}\right]\left[\begin{array}{cc}
\boldsymbol{D}_{S} & 0 \\
0 & 0
\end{array}\right]\left[\begin{array}{cc}
\boldsymbol{J}^{\sim} & -\boldsymbol{L}^{\sim} \boldsymbol{G}_{r} \\
-\boldsymbol{G}_{r} \boldsymbol{K}^{\sim} & \boldsymbol{G}_{n-r} \boldsymbol{M}^{\sim} \boldsymbol{G}_{n-r}
\end{array}\right] \boldsymbol{U}_{R}^{\sim} \\
& =\boldsymbol{U}_{R}\left[\begin{array}{cc}
\boldsymbol{J D}_{S} \boldsymbol{J}^{\sim} & -\boldsymbol{J} \boldsymbol{D}_{S} \boldsymbol{L}^{\sim} \boldsymbol{G}_{r} \\
-\boldsymbol{L} \boldsymbol{D}_{S} \boldsymbol{J}^{\sim} & \boldsymbol{L} \boldsymbol{D}_{S} \boldsymbol{L}^{\sim} \boldsymbol{G}_{r}
\end{array}\right] \boldsymbol{U}_{R}^{\sim}
\end{aligned}
$$

Furthermore, doing some algebra we have,

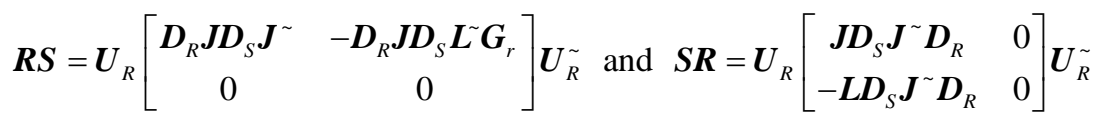

Therefore the equality $\boldsymbol{R S}=\boldsymbol{S} \boldsymbol{R}$, on using the fact that $\boldsymbol{D}_{R}, \boldsymbol{D}_{S}$ and $\boldsymbol{G}_{r}$ are nonsingular, gives

$$
\boldsymbol{D}_{R}\left(\boldsymbol{J} \boldsymbol{D}_{S} \boldsymbol{J}^{\sim}\right)=\left(\boldsymbol{J} \boldsymbol{D}_{S} \boldsymbol{J}^{\sim}\right) \boldsymbol{D}_{R}, \boldsymbol{L} \boldsymbol{D}_{S} \boldsymbol{J}^{\sim}=0 \text { and } \boldsymbol{J} \boldsymbol{D}_{S} \boldsymbol{L}^{\sim}=0
$$

Hence the equivalence follows.

Theorem 3 Let $\boldsymbol{R} \in M_{n}(\mathbb{C})$ be such that $\boldsymbol{R}^{\oplus}$ exists. Then the following statements are equivalent.

1. $\boldsymbol{R}$ is range symmetric.

2. $R(\boldsymbol{R})=R\left(\boldsymbol{R}^{\oplus}\right)$.

3. $N(\boldsymbol{R})=N\left(\boldsymbol{R}^{\oplus}\right)$.

Proof. (i) $\Leftrightarrow$ (ii) Since $\boldsymbol{R}^{\oplus}$ and $\boldsymbol{R}^{\oplus} \boldsymbol{R}$ are m-symmetric idempotents, in fact $\mathrm{m}$ projectors, on using [RS-3], we have $\boldsymbol{R}$ is range symmetric if and only if $\boldsymbol{R} \boldsymbol{R}^{\oplus}=\boldsymbol{R}^{\oplus} \boldsymbol{R}$. Also from [MI-1] and [MI-2] we have $R(\boldsymbol{R})=R\left(\boldsymbol{R} \boldsymbol{R}^{\oplus}\right)$ and $R\left(\boldsymbol{R}^{\oplus}\right)=R\left(\boldsymbol{R}^{\oplus} \boldsymbol{R}\right)$. Therefore $R(\boldsymbol{R})=R\left(\boldsymbol{R}^{\oplus}\right)$. Hence the equivalence follows.

(i) $\Leftrightarrow$ (iii) Similarly $\left(\boldsymbol{I}_{n}-\boldsymbol{R} \boldsymbol{R}^{\oplus}\right)$ and $\left(\boldsymbol{I}_{n}-\boldsymbol{R}^{\oplus} \boldsymbol{R}\right)$ are idempotents such that $N\left(\boldsymbol{R}^{\oplus}\right)=R\left(\boldsymbol{I}_{n}-\boldsymbol{R} \boldsymbol{R}^{\oplus}\right)$ and $N(\boldsymbol{R})=R\left(\boldsymbol{I}_{n}-\boldsymbol{R}^{\oplus} \boldsymbol{R}\right)$. Again using [RS-3], the result follows.

Theorem 4 Let $\boldsymbol{R} \in M_{n}(\mathbb{C})$ be a non zero matrix. Then the following statements are equivalent.

1. $\boldsymbol{R}$ is range symmetric.

2. There exists an invertible matrix $\boldsymbol{M} \in M_{n-r}(\mathbb{C})$ and $\boldsymbol{L} \in M_{(n-r, r)}(\mathbb{C})$ such that 
$\boldsymbol{R}=\boldsymbol{R}^{\sim} \boldsymbol{E} \quad$ with $\quad \boldsymbol{E}=\boldsymbol{U}_{R}\left[\begin{array}{ccc}\left(\boldsymbol{D}_{R}^{\sim}\right)^{-1} & \boldsymbol{D}_{R} & 0 \\ \boldsymbol{L} & & \boldsymbol{M}\end{array}\right] \boldsymbol{U}_{R}^{\sim}$.

3. There exists an invertible matrix $\boldsymbol{M} \in M_{n-r}(\mathbb{C})$ and $\boldsymbol{L} \in M_{(n-r, r)}(\mathbb{C})$ such that $\boldsymbol{R}=\boldsymbol{R}^{\oplus} \boldsymbol{E} \quad$ with $\quad \boldsymbol{E}=\boldsymbol{U}_{R}\left[\begin{array}{cc}\left(\boldsymbol{D}_{R}\right)^{2} & 0 \\ \boldsymbol{L} & \boldsymbol{M}\end{array}\right] \boldsymbol{U}_{R}^{\sim}$.

Proof. (i) $\Leftrightarrow$ (ii) Using [RS-4], there exists an invertible matrix $\boldsymbol{E} \in M_{n}(\mathbb{C})$ such that $\boldsymbol{R}=\boldsymbol{R}^{\sim} \boldsymbol{E}$. We partition $\boldsymbol{E}$ according to the blocks of $\boldsymbol{R}$ such that

$$
\boldsymbol{E}=\boldsymbol{U}_{R}\left[\begin{array}{ll}
\boldsymbol{J} & \boldsymbol{K} \\
\boldsymbol{L} & \boldsymbol{M}
\end{array}\right] \boldsymbol{U}_{R}^{\sim}
$$

Now $\boldsymbol{R}=\boldsymbol{R}^{\sim} \boldsymbol{E}$, gives $\boldsymbol{E}=\boldsymbol{U}_{R}\left[\begin{array}{ccc}\left(\boldsymbol{D}_{R}^{\sim}\right)^{-1} & \boldsymbol{D}_{R} & \mathbf{0} \\ \boldsymbol{L} & & \boldsymbol{M}\end{array}\right] \boldsymbol{U}_{R}^{\sim}$, using the fact that $\boldsymbol{D}_{R}$ is invertible and $\boldsymbol{G}_{r}$ is G-unitary.

(i) $\Leftrightarrow$ (iii) From statement (ii) of the Theorem 3 and [RS-4], we have $R\left(\boldsymbol{R}^{\oplus}\right)=R\left(\boldsymbol{R}^{\sim}\right)$, the equivalence follows on the same lines as above

Theorem 5 Let $\boldsymbol{R} \in M_{n}(\mathbb{C})$ be a nonzero matrix. Then the following statements are equivalent:

1. $\boldsymbol{R}$ is range symmetric.

2. There exists an invertible matrix $\boldsymbol{M} \in M_{n-r}(\mathbb{C})$ and $\boldsymbol{K} \in M_{(r, n-r)}(\mathbb{C})$ such that $\boldsymbol{R}=\boldsymbol{R}^{\sim} \boldsymbol{F} \quad$ with $\boldsymbol{F}=\boldsymbol{U}_{R}\left[\begin{array}{cc}\boldsymbol{D}_{R}\left(\boldsymbol{D}_{R}^{\sim}\right)^{-1} & \boldsymbol{K} \\ 0 & \boldsymbol{M}\end{array}\right] \boldsymbol{U}_{R}^{\sim}$.

3. There exists an invertible matrix $\boldsymbol{M} \in M_{n-r}(\mathbb{C})$ and $\boldsymbol{K} \in M_{(r, n-r)}(\mathbb{C})$ such that $\boldsymbol{R}=\boldsymbol{R}^{\oplus} \boldsymbol{F} \quad$ with $\quad \boldsymbol{F}=\boldsymbol{U}_{R}\left[\begin{array}{cc}\left(\boldsymbol{D}_{R}\right)^{2} & \boldsymbol{K} \\ 0 & \boldsymbol{M}\end{array}\right] \boldsymbol{U}_{R}^{\sim}$.

Proof. The proof follows on the same lines as in the above theorem, using the fact that two matrices $\boldsymbol{R}$ and $\boldsymbol{S}$ are row equivalent if and only if $N(\boldsymbol{R})=N(\boldsymbol{S})$ and utilizing the statement (iii) of Theorem 3 and [RS-2].

\section{Partial Ordering of Range Symmetric Matrices w.r.t Minkowski Adjoint}

In this section some characterizations of predecessors of range symmetric matrices under the partial ordering w.r.t Minkowski adjoint. Using the equivalences of the definition of Partial ordering w.r.t Minkowski adjoint that is [PO-1] and, [PO-2], it can be easily verified that $\boldsymbol{R}^{\sim} \boldsymbol{S}, \boldsymbol{S} \boldsymbol{R}^{\sim}, \boldsymbol{R}^{\oplus} \boldsymbol{S}$ and $\boldsymbol{S} \boldsymbol{R}^{\oplus}$ are m-symmetric.

Theorem 6 Let $\boldsymbol{R}, \boldsymbol{S} \in M_{n}(\mathbb{C})$ such that $S$ is a nonzero range symmetric matrix. Then the following statements are equivalent:

1. $\boldsymbol{R} \leq^{\sim} \boldsymbol{S}$.

2. There exists $\boldsymbol{J} \in M_{r}(\mathbb{C})$ such that 


$$
\boldsymbol{R}=\boldsymbol{U}_{S}\left[\begin{array}{ll}
\boldsymbol{J} & 0 \\
0 & 0
\end{array}\right] \boldsymbol{U}_{S}^{\sim} \quad \text { with } \boldsymbol{J} \leq^{\sim} \boldsymbol{D}_{S}
$$

Proof. (i) $\Leftrightarrow$ (ii) We consider the following block representation of $\boldsymbol{R}$ according to the block size of $S$ as:

$$
\boldsymbol{R}=\boldsymbol{U}_{S}\left[\begin{array}{ll}
\boldsymbol{J} & \boldsymbol{K} \\
\boldsymbol{L} & \boldsymbol{M}
\end{array}\right] \boldsymbol{U}_{S}^{\sim}
$$

Then

$$
\boldsymbol{R}^{\sim} \boldsymbol{R}=\boldsymbol{U}_{S}\left[\begin{array}{cc}
\boldsymbol{J}^{\sim} \boldsymbol{J}-\boldsymbol{L}^{\sim} \boldsymbol{G}_{r} \boldsymbol{L} & \boldsymbol{J}^{\sim} \boldsymbol{K}-\boldsymbol{L}^{\sim} \boldsymbol{G}_{r} \boldsymbol{M} \\
-\boldsymbol{G}_{r} \boldsymbol{K}^{\sim} \boldsymbol{J}+\boldsymbol{G}_{(n-r)} \boldsymbol{M}^{\sim} \boldsymbol{G}_{(n-r)} \boldsymbol{L} & -\boldsymbol{G}_{r} \boldsymbol{K}^{\sim} \boldsymbol{K}+\boldsymbol{G}_{(n-r)} \boldsymbol{M}^{\sim} \boldsymbol{G}_{(n-r)} \boldsymbol{M}
\end{array}\right] \boldsymbol{U}_{S}^{\sim}
$$

and

$$
\boldsymbol{R}^{\sim} \boldsymbol{S}=\boldsymbol{U}_{S}\left[\begin{array}{ll}
\boldsymbol{J} \boldsymbol{D}_{S} & 0 \\
\boldsymbol{L} \boldsymbol{D}_{S} & 0
\end{array}\right] \boldsymbol{U}_{S}^{\sim}
$$

Therefore the equality $\boldsymbol{R}^{\sim} \boldsymbol{R}=\boldsymbol{R}^{\sim} \boldsymbol{S}$ gives $\boldsymbol{J}^{\sim} \boldsymbol{J}-\boldsymbol{L}^{\sim} \boldsymbol{G}_{r} \boldsymbol{L}=\boldsymbol{J} \boldsymbol{D}_{S}$ and $-\boldsymbol{G}_{r} \boldsymbol{K}^{\sim} \boldsymbol{K}+\boldsymbol{G}_{(n-r)} \boldsymbol{M}^{\sim} \boldsymbol{G}_{(n-r)} \boldsymbol{M}=0 \Rightarrow \boldsymbol{K}=0$ and $\boldsymbol{M}=0$ Also computing $\boldsymbol{R R}^{\sim}$ and $\boldsymbol{S} \boldsymbol{R}^{\sim}$ and using the equality $\boldsymbol{R} \boldsymbol{R}^{\sim}=\boldsymbol{S} \boldsymbol{R}^{\sim}$, we get $\boldsymbol{J} \boldsymbol{J}^{\sim}-\boldsymbol{K G}_{r} \boldsymbol{K}^{\sim}=\boldsymbol{D}_{S} \boldsymbol{J}$ and $\boldsymbol{L}=0$. Thus $\boldsymbol{J}^{\sim} \boldsymbol{J}=\boldsymbol{J} \boldsymbol{D}_{S}$ and $\boldsymbol{J} \boldsymbol{J}^{\sim}=\boldsymbol{D}_{S} \boldsymbol{J}$ i.e., $\boldsymbol{J} \leq^{\sim} \boldsymbol{D}_{S}$.

However if $\boldsymbol{S}$ is range symmetric and $\boldsymbol{R} \leq^{\sim} \boldsymbol{S}$, then $\boldsymbol{R}$ need not be range symmetric e.g. consider the matrices

Example 1

$$
\boldsymbol{R}=\left[\begin{array}{ll}
1 & i \\
0 & 0
\end{array}\right] \text { and } \boldsymbol{S}=\left[\begin{array}{cc}
1 & i \\
i & -1
\end{array}\right] \text {, where } i=\sqrt{-1}
$$

Remark 1 If both the matrices $\boldsymbol{R}, \boldsymbol{S} \in M_{n}(\mathbb{C})$ are range symmetric and $\boldsymbol{R} \leq^{\sim} \boldsymbol{S}$, then using the statements [PO-1], [PO-2] and [RS-3], it can be easily observed that $\boldsymbol{R}^{\oplus} \boldsymbol{S}=\boldsymbol{S} \boldsymbol{R}^{\oplus}$. Using the representations (3) and (7) of $\boldsymbol{S}$ and $\boldsymbol{R}$ respectively and Theorem 6.8.3. from [26], we have another equivalent condition for the partial ordering of range symmetric matrices w.r.t minkowski adjoint given by $\boldsymbol{R} \leq^{\sim} \boldsymbol{S} \Leftrightarrow \boldsymbol{R} \boldsymbol{R}^{\oplus}=\boldsymbol{S}^{\oplus} \boldsymbol{R}$ and $\boldsymbol{R}^{\oplus} \boldsymbol{R}=\boldsymbol{R} \boldsymbol{S}^{\oplus}$. Furthermore, $\boldsymbol{R}$ is range symmetric, we have $\boldsymbol{S}^{\oplus} \boldsymbol{R}=\boldsymbol{R} \boldsymbol{S}^{\oplus}$.

The next result gives some equivalent conditions for a matrix $\boldsymbol{R}$ to be range symmetric when $S$ is range symmetric and $S$ is the successor of $\boldsymbol{R}$.

Theorem 7 Let $\boldsymbol{R}, \boldsymbol{S} \in M_{n}(\mathbb{C})$ such that $S$ is a nonzero range symmetric matrix and $\boldsymbol{R} \leq^{\sim} \boldsymbol{S}$, where $\boldsymbol{S}$ is given by (3) and $\boldsymbol{R}$ is given by (7). Then the following statements are equivalent:

1. $\boldsymbol{R}$ is range symmetric.

2. $\boldsymbol{J} \boldsymbol{D}_{S}=\boldsymbol{D}_{S} \boldsymbol{J}$.

3. $\boldsymbol{J}^{\oplus} \boldsymbol{D}_{S}=\boldsymbol{D}_{S} \boldsymbol{J}^{\oplus}$.

4. $\boldsymbol{J}\left(\boldsymbol{J}^{\sim}-\boldsymbol{D}_{S}\right)=\boldsymbol{D}_{S}\left(\boldsymbol{J}^{\sim}-\boldsymbol{J}\right)$.

5. $\left(\boldsymbol{J}^{\sim}-\boldsymbol{D}_{S}\right) \boldsymbol{J}=\left(\boldsymbol{J}^{\sim}-\boldsymbol{J}\right) \boldsymbol{D}_{S}$.

6. $\boldsymbol{J}$ is range symmetric.

Proof. (i) $\Leftrightarrow$ (ii) From remark 1, we have $\boldsymbol{S}^{\oplus} \boldsymbol{R}=\boldsymbol{R} \boldsymbol{S}^{\oplus}$. Now using the facts that 
$\boldsymbol{D}_{S}^{\oplus}=\boldsymbol{D}_{S}^{-1} ; \quad \boldsymbol{D}_{S}$ being invertible and $\boldsymbol{U}_{S}$ is G-unitary and substituting the representations of $\boldsymbol{S}$ and $\boldsymbol{R}$ from (3) and (7) respectively in the above equality and doing some simple algebra leads to $\boldsymbol{J D}_{S}=\boldsymbol{D}_{S} \boldsymbol{J}$

$$
\text { (ii) } \Leftrightarrow \text { (iii) For } \quad \boldsymbol{R}=\boldsymbol{U}_{S}\left[\begin{array}{ll}
\boldsymbol{J} & 0 \\
0 & 0
\end{array}\right] \boldsymbol{U}_{S}^{\sim}, \quad \boldsymbol{R}^{\oplus}=\boldsymbol{U}_{S}\left[\begin{array}{cc}
\boldsymbol{J}^{\oplus} & 0 \\
0 & 0
\end{array}\right] \boldsymbol{U}_{S}^{\sim} \text {. Again using Remark } 1 \text { and }
$$

substituting the respective representations of $\boldsymbol{R}^{\oplus}$ and $S$, the equivalence follows.

(ii) $\Leftrightarrow$ (iv) Using [PO-1] and substituting the representations of $\boldsymbol{R}, \boldsymbol{R}^{\sim}$ and $S$, the equivalence follows after some computation.

On the same lines the equivalences (ii) $\Leftrightarrow$ (v) and (iii) $\Leftrightarrow$ (vi) follow by using the Remark 1 and statements [PO-1] and [PO-2].

The next result similar to Theorem 6 holds if we consider $\boldsymbol{R}$ to be range symmetric and decompose $\boldsymbol{S}$ in terms of representation for $\boldsymbol{R}$

Theorem 8 Let $\boldsymbol{R}, \boldsymbol{S} \in M_{n}(\mathbb{C})$ such that $\boldsymbol{R}$ is a nonzero range symmetric matrix. Then the following statements are equivalent.

1. $\boldsymbol{R} \leq \sim \mathrm{S}$.

2. There exists $\boldsymbol{M} \in M_{n-r}(\mathbb{C})$ such that

$$
\boldsymbol{S}=\boldsymbol{U}_{R}\left[\begin{array}{cc}
\boldsymbol{D}_{R} & 0 \\
0 & \boldsymbol{M}
\end{array}\right] \boldsymbol{U}_{R}^{\sim}
$$

Proof. The proof follows on the same line as in Theorem 6

We again note that if $\boldsymbol{R} \leq \sim \boldsymbol{S}$ and $\boldsymbol{R}$ is range symmetric, then $\boldsymbol{S}$ need not be range symmetric. Consider Example 1 . In the following result we establish some equivalent conditions for $\boldsymbol{S}$ when $\boldsymbol{R}$ is range symmetric and $\boldsymbol{R} \leq \sim \boldsymbol{S}$.

Theorem 9 Let $\boldsymbol{R}, \boldsymbol{S} \in M_{n}(\mathbb{C})$ be given by (2) and (8) respectively such that $\boldsymbol{R}$ is a nonzero range symmetric matrix and $\boldsymbol{R} \leq \sim \boldsymbol{S}$. Then the following statements are equivalent:

1. $S$ is range symmetric.

2. $\boldsymbol{M}$ is range symmetric.

3. $S\left(R^{\oplus}-S^{\oplus}\right)=\left(R^{\oplus}-S^{\oplus}\right) S$.

4. $S^{\oplus}(R-S)=(R-S) S^{\oplus}$.

Proof. (i) $\Leftrightarrow$ (ii) For $\boldsymbol{S}=\boldsymbol{U}_{R}\left[\begin{array}{cc}\boldsymbol{D}_{R} & 0 \\ 0 & \boldsymbol{M}\end{array}\right] \boldsymbol{U}_{R}^{\sim}$, since $\boldsymbol{D}_{R}$ is nonsingular and $\boldsymbol{U}_{R}$ is Gunitary, direct verification shows that $\boldsymbol{S}^{\oplus}=\boldsymbol{U}_{R}\left[\begin{array}{cc}\boldsymbol{D}_{R}^{\oplus} & 0 \\ 0 & \boldsymbol{M}^{\oplus}\end{array}\right] \boldsymbol{U}_{R}^{\sim}$. Therefore $\boldsymbol{S S}^{\oplus}=\boldsymbol{U}_{R}\left[\begin{array}{cc}\boldsymbol{I}_{r} & 0 \\ 0 & \boldsymbol{M} \boldsymbol{M}^{\oplus}\end{array}\right] \boldsymbol{U}_{R}^{\sim} \quad$ and $\quad \boldsymbol{S}^{\oplus} \boldsymbol{S}=\boldsymbol{U}_{R}\left[\begin{array}{cc}\boldsymbol{I}_{r} & 0 \\ 0 & \boldsymbol{M}^{\oplus} \boldsymbol{M}\end{array}\right] \boldsymbol{U}_{R}^{\sim} . \quad \boldsymbol{S}$ being range symmetric, by [RS-3] we have $\boldsymbol{S S}^{\oplus}=\boldsymbol{S}^{\oplus} \boldsymbol{S}$. This gives $\boldsymbol{M} \boldsymbol{M}^{\oplus}=\boldsymbol{M}^{\oplus} \boldsymbol{M}$ and the equivalence follows.

(i) $\Rightarrow$ (iii) Since $\boldsymbol{R} \leq^{\sim} \boldsymbol{S}$ and $\boldsymbol{R}$ and $\boldsymbol{S}$ are range symmetric, using the observation mentioned in Remark 1 i.e., $\boldsymbol{R}^{\oplus} \boldsymbol{S}=\boldsymbol{S} \boldsymbol{R}^{\oplus}$, we have $\boldsymbol{S} \boldsymbol{R}^{\oplus}-\boldsymbol{S} \boldsymbol{S}^{\oplus}=\boldsymbol{R}^{\oplus} \boldsymbol{S}-\boldsymbol{S}^{\oplus} \boldsymbol{S}$, the equivalence follows.

(iii) $\Rightarrow\left(\right.$ i) Since $\boldsymbol{R} \leq \sim \boldsymbol{S}$ and $\boldsymbol{R}$ is range symmetric, again by the same fact that $\boldsymbol{R}^{\oplus}$ 
and $S$ commute, using (iii) i.e., $S\left(R^{\oplus}-S^{\oplus}\right)=\left(R^{\oplus}-S^{\oplus}\right) S$, we get $S$ is range symmetric.

(i) $\Leftrightarrow$ (iv) From Remark 1, we have $\boldsymbol{R S}^{\oplus}=\boldsymbol{S}^{\oplus} \boldsymbol{R}$. This gives $\boldsymbol{R} \boldsymbol{S}^{\oplus}-\boldsymbol{S} \boldsymbol{S}^{\oplus}=\boldsymbol{S}^{\oplus}-\boldsymbol{S} \boldsymbol{S}^{\oplus} \boldsymbol{R}$. Now using the fact that $S$ is range symmetric the equivalence follows.

In the above results we have used the commutativity of $\boldsymbol{R}$ and $\boldsymbol{S}^{\oplus}$ and $\boldsymbol{R}^{\oplus}$ and $S$. However if we assume the conditions given in the above theorem with an additional assumption that $\boldsymbol{R S}=\boldsymbol{S R}$, then the conditions obtained by interchanging $\boldsymbol{R}$ and $\boldsymbol{S}$ are also equivalent.

Theorem 10 Let $\boldsymbol{R}, \boldsymbol{S} \in M_{n}(\mathbb{C})$ be range symmetric such that $\boldsymbol{R}$ is a non zero matrix. Then the following statements are equivalent.

1. $\boldsymbol{R} \leq \sim \mathrm{S}$.

2. There exists a G-unitary matrix $\boldsymbol{U} \in M_{n}(\mathbb{C}), \quad \boldsymbol{D} \in M_{r}(\mathbb{C})$ and $\boldsymbol{M} \in M_{r-s}(\mathbb{C})$ such that $\boldsymbol{R}=\boldsymbol{U}\left[\begin{array}{lll}\boldsymbol{D} & 0 & 0 \\ 0 & 0 & 0 \\ 0 & 0 & 0\end{array}\right] \boldsymbol{U}^{\sim}$ and $\boldsymbol{S}=\boldsymbol{U}\left[\begin{array}{ccc}\boldsymbol{D} & 0 & 0 \\ 0 & \boldsymbol{M} & 0 \\ 0 & 0 & 0\end{array}\right] \boldsymbol{U}^{\sim}$

Proof. (i) $\Rightarrow$ (ii) Consider the decomposition of $S$ given by (3) i.e., $\boldsymbol{S}=\boldsymbol{U}_{S}\left[\begin{array}{cc}\boldsymbol{D}_{S} & 0 \\ 0 & 0\end{array}\right] \boldsymbol{U}_{S}^{\sim}$. Since $\boldsymbol{S}$ is range symmetric, therefore by Theorem 6 , there exists $\boldsymbol{J} \in M_{r}(\mathbb{C})$ such that $\boldsymbol{R}=\boldsymbol{U}_{S}\left[\begin{array}{ll}\boldsymbol{J} & 0 \\ 0 & 0\end{array}\right] \boldsymbol{U}_{S}^{\sim}$ with $\boldsymbol{J} \leq^{\sim} \boldsymbol{D}_{s}$. Using Theorem 7, we have $\boldsymbol{J}$ is range symmetric. We consider the following block representation of $\boldsymbol{J}$ as $\boldsymbol{J}=\boldsymbol{U}_{J}\left[\begin{array}{cc}\boldsymbol{D}_{J} & 0 \\ 0 & 0\end{array}\right] \boldsymbol{U}_{J}^{\sim}$, where $\boldsymbol{U}_{J}$ is G-unitary and $\boldsymbol{D} \in M_{r}(\mathbb{C})$ is invertible. Since $\boldsymbol{J} \leq^{\sim} \boldsymbol{D}_{S}$, by Theorem 8, we can find $\boldsymbol{M} \in M_{r-s}(\mathbb{C})$ such that $\boldsymbol{D}_{S}=\boldsymbol{U}_{J}\left[\begin{array}{cc}\boldsymbol{D}_{J} & 0 \\ 0 & \boldsymbol{M}\end{array}\right] \boldsymbol{U}_{J}^{\sim}$.

Thus $\boldsymbol{M}$ is nonsingular when $\boldsymbol{M} \neq 0$. Taking $\boldsymbol{D}_{J}=\boldsymbol{D}$, we have $\boldsymbol{S}=\boldsymbol{U}\left[\begin{array}{ccc}\boldsymbol{D} & 0 & 0 \\ 0 & \boldsymbol{M} & 0 \\ 0 & 0 & 0\end{array}\right] \boldsymbol{U}^{\sim}$, where $\boldsymbol{U}=\boldsymbol{U}_{S}\left[\begin{array}{cc}\boldsymbol{D}_{J} & 0 \\ 0 & \boldsymbol{I}\end{array}\right]$ is G-unitary.

$(i) \Rightarrow(i i)$ Follows at once by direct verification.

\section{Partial Ordering of M-Projectors}

In this section we obtain some results on partial ordering of m-projectors w.r.t Minkowski adjoint. The following result from [5], with two more obvious conditions, will be used extensively in the forthcoming results.

Lemma 1 Let $R \in \mathcal{M}$ be range symmetric, then

1. $\boldsymbol{R}^{\oplus}=\left(\boldsymbol{R}+\tilde{\boldsymbol{P}}_{R}\right)^{-1}\left(\boldsymbol{I}_{n}-\tilde{\boldsymbol{P}}_{R}\right)$.

2. $\tilde{\boldsymbol{P}}_{R}$ is idempotent.

3. $\boldsymbol{R} \tilde{\boldsymbol{P}}_{R}=\tilde{\boldsymbol{P}}_{R} \boldsymbol{R}=\boldsymbol{R}^{\oplus} \tilde{\boldsymbol{P}}_{R}=\tilde{\boldsymbol{P}}_{R} \boldsymbol{R}^{\oplus}=0$.

4. $\tilde{\boldsymbol{P}}_{R}=0$ if and only if $\boldsymbol{R}$ is nonsingular.

5. $\boldsymbol{R}=\boldsymbol{P}(\boldsymbol{D} \oplus 0) \boldsymbol{P}^{\sim}$ then $\tilde{\boldsymbol{P}}_{R}=\boldsymbol{P}\left(0 \oplus \boldsymbol{I}_{n-r}\right) \boldsymbol{P}^{\sim}$. 
6. $\operatorname{rank}\left(\tilde{\boldsymbol{P}}_{R}\right)=n-\operatorname{rank}(\boldsymbol{R})$.

7. $\tilde{\boldsymbol{P}}_{R}$ is invertible then $\boldsymbol{R}=0$ and if $\boldsymbol{R}=0$, then $\tilde{\boldsymbol{P}}_{R}=\boldsymbol{I}_{n}$.

8. $\tilde{\boldsymbol{P}}_{R}$ has index atmost one.

Lemma 2 Let $\boldsymbol{R}, \boldsymbol{S} \in M_{n}^{0} \cup M_{n}^{1}$. Then

1. If $\boldsymbol{R} \leq^{\sim} \boldsymbol{S}$, then $\operatorname{rk}(\boldsymbol{R}) \leq \operatorname{rk}(\boldsymbol{S})$.

2. $\boldsymbol{R} \leq \sim \tilde{\boldsymbol{P}}_{R} \Leftrightarrow \boldsymbol{R}=0$.

3. $\tilde{\boldsymbol{P}}_{R} \leq \sim \boldsymbol{R} \Leftrightarrow \boldsymbol{R}$ is invertible.

4. If $\boldsymbol{R}$ is nonzero singular matrix then $\boldsymbol{R}$ and $\tilde{\boldsymbol{P}}_{R}$ are incomparable under the partial ordering w.r.t Minkowski adjoint.

5. $\tilde{\boldsymbol{P}}_{S} \leq \sim \tilde{\boldsymbol{P}}_{R}$, then $\operatorname{rk}(\boldsymbol{R}) \leq \operatorname{rk}(\boldsymbol{S})$.

6. $\boldsymbol{R} \leq \sim \boldsymbol{R}+\tilde{\boldsymbol{P}}_{R}$.

Proof. (i) Since $R(\boldsymbol{R})=R\left(\boldsymbol{R} \boldsymbol{R}^{\oplus} \boldsymbol{R}\right) \subseteq R\left(\boldsymbol{R} \boldsymbol{R}^{\oplus}\right)=R\left(\boldsymbol{S} \boldsymbol{R}^{\oplus}\right) \subseteq R(\boldsymbol{S})$. This gives $\operatorname{rk}(\boldsymbol{R}) \leq \operatorname{rk}(\boldsymbol{S})$.

(ii) $\boldsymbol{R} \leq^{\sim} \tilde{\boldsymbol{P}}_{R}$, then $\boldsymbol{R} \boldsymbol{R}^{\oplus} \boldsymbol{R}=\boldsymbol{R}^{\oplus} \tilde{\boldsymbol{P}}_{R}=0 \Rightarrow \boldsymbol{R}=0$. Conversely if $\boldsymbol{R}=0, \boldsymbol{R}^{\oplus}=0$ and $\tilde{\boldsymbol{P}}_{R}=\boldsymbol{I}_{n}$ and hence $\boldsymbol{R} \leq \sim \tilde{\boldsymbol{P}}_{R}$.

(iii) From statement (ii) of Lemma 1 and the fact that $\left(\tilde{\boldsymbol{P}}_{R}\right)^{\oplus}=\tilde{\boldsymbol{P}}_{R}$, if $\tilde{\boldsymbol{P}}_{R} \leq \sim \boldsymbol{R}$, then $\tilde{\boldsymbol{P}}_{R}=\tilde{\boldsymbol{P}}_{R} \boldsymbol{R}=0$ and hence by point (iv) of Lemma $1 \boldsymbol{R}$ is invertible. Again by the same argument i.e, point (iv) of Lemma 1 converse holds.

(iv) It is obvious from (ii) and (iii).

(v) Follows at once by using point (i) of the Lemma 2 and point (vi) of Lemma 1.

(vi) The statement follows at once on using the fact that $\boldsymbol{R}^{\oplus} \tilde{\boldsymbol{P}}_{R}=0$.

Lemma 3 Let $\boldsymbol{R} \in M_{n}^{0} \cup M_{n}^{1}$. Then

1. If $\boldsymbol{R}$ is range symmetric, then $\tilde{\boldsymbol{P}}_{R}$ is $\mathrm{m}$-symmetric and hence range symmetric

2. If $\tilde{\boldsymbol{P}}_{R}$ is range symmetric, then so is $\boldsymbol{R}$

Proof. (i) The statement follows at once on using the [RS-3], [MI-3] and [MI-4].

(ii) If $\tilde{\boldsymbol{P}}_{R}=0$, the the result is trivial. Let $\tilde{\boldsymbol{P}}_{R} \neq 0$ such that $\operatorname{rk}\left(\tilde{\boldsymbol{P}}_{R}\right)=r$, then by point (v) of Lemma 1 we have $\tilde{\boldsymbol{P}}_{R}=\boldsymbol{U}\left[\begin{array}{cc}\boldsymbol{D} & 0 \\ 0 & 0\end{array}\right] \boldsymbol{U}^{\sim}$. Also using point (ii) of Lemma 1 we get $\boldsymbol{D}^{2}=\boldsymbol{D}$ i.e., $\boldsymbol{D}=\boldsymbol{I}_{r}$. Thus we have $\boldsymbol{R}^{\oplus}=\boldsymbol{U}\left[\begin{array}{cc}0 & 0 \\ 0 & \boldsymbol{I}_{n-r}\end{array}\right] \boldsymbol{U}^{\sim}$. Consider the block representation of $\boldsymbol{R}$, where the partition is done according to the blocks of $\tilde{\boldsymbol{P}}_{R}$ such that $\boldsymbol{R}=\boldsymbol{U}\left[\begin{array}{ll}\boldsymbol{J} & \boldsymbol{K} \\ \boldsymbol{L} & \boldsymbol{M}\end{array}\right] \boldsymbol{U}^{\sim}$. Using [MI-1] and [MI-2], we get $\boldsymbol{J}, \boldsymbol{K}$ and $\boldsymbol{L}=0$. Therefore $\boldsymbol{R}^{\oplus}=\boldsymbol{U}\left[\begin{array}{cc}0 & 0 \\ 0 & \boldsymbol{M} \boldsymbol{M}^{\oplus}\end{array}\right] \boldsymbol{U}^{\sim}$. This shows that $\boldsymbol{M}$ is nonsingular and the result follows.

Remark 2 Since $\tilde{\boldsymbol{P}}_{R}$ is a m-projector [5], we have $\left(\tilde{\boldsymbol{P}}_{R}\right)^{\oplus}=\tilde{\boldsymbol{P}}_{R}$. If we write $\tilde{\boldsymbol{P}}_{R}=\tilde{\boldsymbol{P}}_{1}(\boldsymbol{R})$ i.e., we take $\tilde{\boldsymbol{P}}$ as a function of $\boldsymbol{R}$, then $\tilde{\boldsymbol{P}}_{2}(\boldsymbol{R})=\tilde{\boldsymbol{P}}\left(\tilde{\boldsymbol{P}}_{1}(\boldsymbol{R})\right)=\boldsymbol{I}_{n}-\tilde{\boldsymbol{P}}_{R}\left(\tilde{\boldsymbol{P}}_{R}\right)^{\oplus}=\boldsymbol{R} \boldsymbol{R}^{\oplus}=0 \Leftrightarrow \boldsymbol{R}=0$. Thus $\tilde{\boldsymbol{P}}_{2}(0)=0$. However $\tilde{\boldsymbol{P}}_{2}(\boldsymbol{R}) \neq \boldsymbol{R}$ in general. Consider the decomposition $\boldsymbol{R}=\boldsymbol{U}\left[\begin{array}{cc}\boldsymbol{D}_{R} & 0 \\ 0 & 0\end{array}\right] \boldsymbol{U}^{\sim}$, we have 
$\tilde{\boldsymbol{P}}_{2}(\boldsymbol{R})=\boldsymbol{R} \Leftrightarrow \boldsymbol{D}_{R}=\boldsymbol{I}_{r}$ i.e, $\boldsymbol{R}=\boldsymbol{U}\left[\begin{array}{cc}\boldsymbol{I}_{r} & 0 \\ 0 & 0\end{array}\right] \boldsymbol{U}^{\sim}$, which is the fundamental representation of a m-projector. Hence we conclude that $\tilde{\boldsymbol{P}}_{2}(\boldsymbol{R})=\boldsymbol{R}$ if and only if $\boldsymbol{R}$ is a $\mathrm{m}$ projector.

We generalize the function $\tilde{\boldsymbol{P}}(\boldsymbol{R})$ by defining it as:

$$
\tilde{\boldsymbol{P}}_{k}(\boldsymbol{R})=\left(\tilde{\boldsymbol{P}}_{k-1} \tilde{\boldsymbol{P}}_{1}\right)(\boldsymbol{R})=\tilde{\boldsymbol{P}}_{k-1}\left(\tilde{\boldsymbol{P}}_{1}(\boldsymbol{R})\right)
$$

Thus we have the following equations

$$
\tilde{\boldsymbol{P}}_{k}(\boldsymbol{R})= \begin{cases}\tilde{\boldsymbol{P}}_{1}(\boldsymbol{R}) & \text { if } k \text { is odd } \\ \boldsymbol{R} \boldsymbol{R}^{\oplus} & \text { if } k \text { is even }\end{cases}
$$

and hence if $\mathrm{R}=0$, we get

$$
\tilde{\boldsymbol{P}}_{k}(\boldsymbol{R})= \begin{cases}\boldsymbol{I}_{n} & \text { if } k \text { is odd } \\ 0 & \text { if } k \text { is even }\end{cases}
$$

Let us consider some sets with following notations:

$$
\begin{gathered}
\Gamma=\{\boldsymbol{R} \in \mathcal{M}: \boldsymbol{R} \text { is range symmetric }\} \\
\Delta=\left\{\boldsymbol{R} \in M_{n}^{0} \cup M_{n}^{1}: \tilde{\boldsymbol{P}}_{1}(\boldsymbol{R}) \text { is range symmetric }\right\}
\end{gathered}
$$

and

$$
\Lambda=\left\{\boldsymbol{R} \in M_{n}^{0} \cup M_{n}^{1}: \tilde{\boldsymbol{P}}_{1}(\boldsymbol{R}) \text { is range symmetric and } \tilde{\boldsymbol{P}}_{1}(\boldsymbol{R}) \neq 0\right\}
$$

Theorem 11 Let $\Gamma, \Delta$ and $\Lambda$ be the sets defined in (12), (13) and(14) respectively. Then, $\Gamma=\Delta$ and $\Gamma \cap M_{n}^{1}=\Lambda$.

Proof. The proof follows easily by utilizing Lemmas 1 and 3 .

From the statement (i) of Lemma 3 , it is obvious that $\tilde{\boldsymbol{P}}_{1}(\Gamma) \subseteq \Gamma$. However the reverse inclusion does not hold in general. Consider the matrix $R=\left[\begin{array}{ll}4 & 0 \\ 0 & 0\end{array}\right]$. If possible suppose there exist a matrix $\boldsymbol{J} \in M_{2}(\mathbb{C}) \cap \Gamma$ such that $\boldsymbol{R}=\tilde{\boldsymbol{P}}_{1}(\boldsymbol{J})$, then by Lemma 3, we have $\boldsymbol{J}=\boldsymbol{U}\left[\begin{array}{ll}0 & 0 \\ 0 & 1\end{array}\right] \boldsymbol{U}^{\sim}$, which is absurd, since $4 \in \sigma(\boldsymbol{R})$ but $4 \notin \sigma(\boldsymbol{J})$. Therefore $\Gamma \not \subset \tilde{\boldsymbol{P}}_{1}(\Gamma)$.

Remark 3 Let $\boldsymbol{R} \in M_{n}^{1}-\{0\}$, then by using Lemma $1 \quad \tilde{\boldsymbol{P}}_{1}(\boldsymbol{R}) \subseteq M_{n}^{1}-\{0\}$. If $\boldsymbol{R} \in \Lambda-\{0\}$, then by Remark 2, we have $\tilde{\boldsymbol{P}}_{1}(\boldsymbol{R}) \in \mathbb{C}_{n}^{m p} \cap M_{n}^{1}-\{0\}=\left(\mathbb{C}_{n}^{m p} \cap \Lambda\right)-\{0\}$ and if $\boldsymbol{R} \in \Lambda$, then by Theorem 11, we have $\tilde{\boldsymbol{P}}_{1}(\boldsymbol{R}) \in\left[\left(\mathbb{C}_{n}^{m p} \cap \Lambda\right)-\{0\}\right] \cup\left\{\boldsymbol{I}_{n}\right\}$.

The next result provides a characterization of the set

$$
\Psi=\left\{\boldsymbol{S} \in M_{n}^{0} \cup M_{n}^{1}: 0 \leq^{\sim} \boldsymbol{S} \leq \sim \tilde{\boldsymbol{P}}_{1}(\boldsymbol{S})\right\}
$$

Theorem 12 Let $\boldsymbol{R} \in M_{n}(\mathbb{C})$ be range symmetric given by (2), then

$$
\Psi=\left\{\boldsymbol{U}_{R}(0 \oplus \boldsymbol{M}) \boldsymbol{U}_{R}^{\sim}: \boldsymbol{M} \in \mathbb{C}_{n-r}^{m p}\right\} \subseteq \mathbb{C}_{n}^{m p}
$$


Proof. Let $\boldsymbol{R}=\boldsymbol{U}\left[\begin{array}{cc}\boldsymbol{D}_{R} & 0 \\ 0 & 0\end{array}\right] \boldsymbol{U}^{\sim}$. Then $\tilde{\boldsymbol{P}}_{1}(\boldsymbol{R})=\boldsymbol{U}_{R}\left[\begin{array}{cc}0 & 0 \\ 0 & \boldsymbol{I}_{n-r}\end{array}\right] \boldsymbol{U}_{R}^{\sim}$. Therefore for $\boldsymbol{S} \leq^{\sim} \boldsymbol{R}$, we have $\boldsymbol{S}=\boldsymbol{U}_{R}\left[\begin{array}{cc}0 & 0 \\ 0 & \boldsymbol{M}\end{array}\right] \boldsymbol{U}_{R}^{\sim}$ such that $\boldsymbol{M} \leq^{\sim} \boldsymbol{I}_{n-r}$ i.e., $\boldsymbol{M}^{2}=\boldsymbol{M}=\boldsymbol{M}^{\sim}$ and the result follows.

The next result shows that the function $\tilde{\boldsymbol{P}}_{1}(\boldsymbol{R})$, when restricted to the set $\Gamma$ is monotonically decreasing w.r.t the partial ordering w.r.t Minkowski adjoint.

Theorem 13 Let $\boldsymbol{R}, \boldsymbol{S} \in \Gamma$, such that $\boldsymbol{R} \leq \sim \boldsymbol{S}$. Then $\tilde{\boldsymbol{P}}_{1}(\boldsymbol{R}) \leq^{\sim} \tilde{\boldsymbol{P}}_{1}(\boldsymbol{S})$

Proof. Let $\boldsymbol{R}, \boldsymbol{S} \in \Gamma$, such that $\boldsymbol{R} \leq \sim \boldsymbol{S}$. Since $\boldsymbol{R}$ is range symmetric we have

$$
\boldsymbol{R}=\boldsymbol{R} \boldsymbol{R}^{\oplus} \boldsymbol{R}=\boldsymbol{R}^{2} \boldsymbol{R}^{\oplus}=\boldsymbol{R}^{\oplus} \boldsymbol{R}^{2}
$$

and therefore

$$
\boldsymbol{R S}=\boldsymbol{R}^{2} \boldsymbol{R}^{\oplus} \boldsymbol{S}=\boldsymbol{R}^{2} \boldsymbol{R}^{\oplus} \boldsymbol{R}=\boldsymbol{R}^{2}=\boldsymbol{R} \boldsymbol{R}^{\oplus} \boldsymbol{R}^{2}=\boldsymbol{S} \boldsymbol{R}^{\oplus} \boldsymbol{R}^{2}=\boldsymbol{S} \boldsymbol{R}
$$

Also

$$
\boldsymbol{R} \boldsymbol{R}^{\oplus} \boldsymbol{S} \boldsymbol{S}^{\oplus}=\boldsymbol{R S} \boldsymbol{R}^{\oplus} \boldsymbol{S}^{\oplus}=S R \boldsymbol{R}^{\oplus} \boldsymbol{S}^{\oplus}=S \boldsymbol{R}^{\oplus} \boldsymbol{R} \boldsymbol{S}^{\oplus}=S \boldsymbol{S}^{\oplus} \boldsymbol{R} \boldsymbol{S}^{\oplus}=S \boldsymbol{S}^{\oplus} \boldsymbol{R} \boldsymbol{R}^{\oplus}
$$

Finally using (15) and (16) we get $\tilde{\boldsymbol{P}}_{1}(\boldsymbol{S})=\tilde{\boldsymbol{P}}_{1}(\boldsymbol{R}) \tilde{\boldsymbol{P}}_{1}(\boldsymbol{S})$ and $\tilde{\boldsymbol{P}}_{1}(\boldsymbol{S})=\tilde{\boldsymbol{P}}_{1}(\boldsymbol{S}) \tilde{\boldsymbol{P}}_{1}(\boldsymbol{R})$. Hence $\tilde{\boldsymbol{P}}_{1}(\boldsymbol{R}) \leq \sim \tilde{\boldsymbol{P}}_{1}(\boldsymbol{S})$.

However for the range symmetric matrices $\boldsymbol{R}=\left[\begin{array}{ll}1 & 0 \\ 0 & 0\end{array}\right]$ and $\boldsymbol{S}=\left[\begin{array}{ll}2 & 0 \\ 0 & 0\end{array}\right]$, we have $\tilde{\boldsymbol{P}}_{1}(\boldsymbol{R}) \leq^{\sim} \tilde{\boldsymbol{P}}_{1}(\boldsymbol{S})$ but $\boldsymbol{S}^{\sim} \boldsymbol{R}$. Thus we have the following result.

Theorem 14 Let $\boldsymbol{R}, \boldsymbol{S} \in \mathbb{C}_{n}^{m p}$, such that $\tilde{\boldsymbol{P}}_{1}(\boldsymbol{S}) \leq^{\sim} \tilde{\boldsymbol{P}}_{1}(\boldsymbol{R})$. Then $\boldsymbol{R} \leq^{\sim} \boldsymbol{S}$.

Proof. The proof follows at once by using Theorem 13 and Remark 2.

Theorem 15 Let $\boldsymbol{R}$ be range symmetric and $\boldsymbol{S} \in M_{n}^{0} \cup M_{n}^{1}$ be such that $\boldsymbol{R} \leq^{\sim} \boldsymbol{S}$. Then $\tilde{\boldsymbol{P}}_{1}(\boldsymbol{S}) \leq \sim \tilde{\boldsymbol{P}}_{1}(\boldsymbol{R}) \Leftrightarrow \tilde{\boldsymbol{P}}_{1}(\boldsymbol{S}) \in \mathbb{C}_{n}^{m p}$.

Proof. Consider the decomposition of $\boldsymbol{R}$ as given in (2). Then from Theorem 8 we get $\boldsymbol{S}=\boldsymbol{U}\left[\begin{array}{cc}\boldsymbol{D}_{R} & 0 \\ 0 & \boldsymbol{M}\end{array}\right]$ and hence $\tilde{\boldsymbol{P}}_{1}(\boldsymbol{S})=\left[\begin{array}{cc}0 & 0 \\ 0 & \tilde{\boldsymbol{P}}_{1}(\boldsymbol{M})\end{array}\right]$. Thus if we assume $\tilde{\boldsymbol{P}}_{1}(\boldsymbol{S}) \leq \sim \tilde{\boldsymbol{P}}_{1}(\boldsymbol{R})$, then from Theorem 6, we get $\tilde{\boldsymbol{P}}_{1}(\boldsymbol{S})=\left[\begin{array}{ll}0 & 0 \\ 0 & \boldsymbol{J}\end{array}\right]$ with $\boldsymbol{J} \leq^{\sim} \boldsymbol{I}_{n-r}$. Therefore $\boldsymbol{J}=\tilde{\boldsymbol{P}}_{1}(\boldsymbol{M})$. Hence $\tilde{\boldsymbol{P}}_{1}(\boldsymbol{S}) \in \mathbb{C}_{n}^{m p}$, Conversely if we assume that $\tilde{\boldsymbol{P}}_{1}(\boldsymbol{S}) \in \mathbb{C}_{n}^{m p}$, then $S$ is range symmetric and finally from Theorem 13, the result follows.

Theorem 16 Let $\boldsymbol{R} \neq 0 \in M_{n}^{0} \cup M_{n}^{1}$ has the representation as given in point $(v)$ of Lemma 1 and $\boldsymbol{S} \in M_{n}^{0} \cup M_{n}^{1}$. Then $\tilde{\boldsymbol{P}}_{1}(\boldsymbol{R}) \leq^{\sim} \tilde{\boldsymbol{P}}_{1}(\boldsymbol{S})$ if and only if there exists $\boldsymbol{E} \in M_{n}^{0} \cup M_{n}^{1}$ such that $\boldsymbol{S}=\boldsymbol{P}\left[\begin{array}{ll}\boldsymbol{E} & 0 \\ 0 & 0\end{array}\right] \boldsymbol{P}^{\sim}$.

Proof. Assume that $\tilde{\boldsymbol{P}}_{1}(\boldsymbol{R}) \leq \sim \tilde{\boldsymbol{P}}_{1}(\boldsymbol{S})$. Then from Theorem 13 we have $\tilde{\boldsymbol{P}}_{1}(\boldsymbol{S}) \tilde{\boldsymbol{P}}_{1}(\boldsymbol{R})=\tilde{\boldsymbol{P}}_{1}(\boldsymbol{R})=\tilde{\boldsymbol{P}}_{1}(\boldsymbol{R}) \tilde{\boldsymbol{P}}_{1}(\boldsymbol{S})$. Let $\tilde{\boldsymbol{P}}_{1}(\boldsymbol{S})=\boldsymbol{P}\left[\begin{array}{ll}\boldsymbol{J} & \boldsymbol{K} \\ \boldsymbol{L} & \boldsymbol{M}\end{array}\right] \boldsymbol{P}^{\sim}$, where the blocks are partitioned according to the blocks of $\boldsymbol{R}$. Using the above mentioned equality we have 
$\boldsymbol{K}=0, \quad \boldsymbol{L}=0$ and $\boldsymbol{M}=\boldsymbol{I}_{n-r}$ and hence $\tilde{\boldsymbol{P}}_{1}(\boldsymbol{S})=\boldsymbol{P}\left[\begin{array}{cc}\boldsymbol{J} & 0 \\ 0 & \boldsymbol{I}_{n-r}\end{array}\right] \boldsymbol{P}^{\sim}$ Also $\boldsymbol{S S}^{\oplus}=\boldsymbol{S}^{\oplus} \boldsymbol{S}=\boldsymbol{I}_{n}-\tilde{\boldsymbol{P}}_{1}(\boldsymbol{S})=\boldsymbol{P}\left[\begin{array}{cc}\boldsymbol{I}_{r}-\boldsymbol{J} & 0 \\ 0 & 0\end{array}\right] \boldsymbol{P}^{\sim}$. Taking $\boldsymbol{S}=\boldsymbol{P}\left[\begin{array}{ll}\boldsymbol{E} & \boldsymbol{F} \\ \boldsymbol{G} & \boldsymbol{H}\end{array}\right] \boldsymbol{P}^{\sim}$, where the decomposition is done according to the blocks of $\tilde{\boldsymbol{P}}_{1}(\boldsymbol{S})$. Then from the equation $\boldsymbol{S}=\left(\boldsymbol{S} \boldsymbol{S}^{\oplus}\right) \boldsymbol{S}=\boldsymbol{S}\left(\boldsymbol{S}^{\oplus} \boldsymbol{S}\right)$ we get $\boldsymbol{G}=0, \boldsymbol{H}=0, \boldsymbol{F}=0, \boldsymbol{J} \boldsymbol{E}=0=\boldsymbol{E} \boldsymbol{J}$ and $\boldsymbol{J} \boldsymbol{F}=0$ and therefore $\boldsymbol{S}=\boldsymbol{P}\left[\begin{array}{ll}\boldsymbol{E} & 0 \\ 0 & 0\end{array}\right] \boldsymbol{P}^{\sim}$ with $\boldsymbol{J} \boldsymbol{E}=0=\boldsymbol{E} \boldsymbol{J}$. Clearly $\boldsymbol{E} \in M_{r}(\mathbb{C})$ has index one. The converse is obvious.

\section{Acknowledgements}

The second author was supported by UGC-BSR through grant No. F25-1/2014-15(BSR)/ 7-254/2009(BSR) (20.01.2015). This support is greatly appreciated.

\section{References}

[1] Gohberg, I., Lancaster, P. and Rodman, L. (2005) Indefinite Linear Algebra and Applications. Birkhäuser, Verlag, Basel, Boston, Berlin.

[2] Meenakshi, A.R. (2000) Generalized Inverse of Matrices in Minkowski Space. Proceedings of National Seminar on Algebra and Its Applications, 1, 1-14.

[3] Meenakshi, A.R. (2000) Range Symmetric Matrices in Minkowski Space. Bulletin of the Malaysian Mathematical Sciences Society, 23, 45-52.

[4] Meenakshi, A.R. and Krishnaswamy, D. (2006) Product of Range Symmetric Block Matrices in Minkowski Space. Bulletin of the Malaysian Mathematical Sciences Society, 29, 59-68.

[5] Lone, M.S. and Krishnaswamy, D. (2016) m-Projections Involving Minkowski Inverse and Range Symmetric Property in Minkowski Space. Journal of Linear and Topological Algebra.

[6] Krishnaswamy, D. (2005) Contributions to the Study on Range Symmetric Matrices in Minkowski Space. Ph.D. Dissertation, Annamalai University, India.

[7] Ben-Isreal, A. and Greville, T. (2003) Generalized Inverse: Theory and Applications. 2nd Edition, Springer Verlag, New York.

[8] Campbell, S.L. and Meyer Jr., C.D. (1991) Generalized Inverse of Linear Transformations. 2nd Edition, Dover, New York.

[9] Prasolov, V.V. (1994) Problems and Theorems in Linear Algebra. American Mathematical Society, Providence.

[10] Meyer, C.D. (2000) Matrix Analysis and Applied Linear Algebra. SIAM, Philadelphia. https://doi.org/10.1137/1.9780898719512

[11] Mitra, S.K., Bhimasankaram, P. and Malik, S.B. (2010) Matrix Partial Orders, Shorted Operators and Applications. World Scientific Publishing Company, Singapore.

[12] Rao, C.R. and Mitra, S.K. (1971) Generalized Inverse of Matrices and Its Applications. John Wiley \& Sons, New York.

[13] Tosic, M. and Cvetkovic-Ilic, D.S. (2012) Invertibility of a Linear Combination of Two Matrices and Partial Orderings. Applied Mathematics and Computation, 218, 4651-4657. https://doi.org/10.1016/j.amc.2011.10.052

[14] Malik, S.B. (2013) Some More Properties of Core Partial Order. Applied Mathematics and 
Computation, 221, 192-201. https://doi.org/10.1016/j.amc.2013.06.012

[15] Malik, S.B., Ruedab, L. and Thome, N. (2014) Further Properties on the Core Partial Order and Other Matrix Partial Orders. Linear Multilinear Algebra, 62, 1629-1648. https://doi.org/10.1080/03081087.2013.839676

[16] Baksalary, J.K. and Mitra, S.K. (1991) Left-Star and Right-Star Partial Orderings. Linear Algebra and Its Applications, 149, 73-89. https://doi.org/10.1016/0024-3795(91)90326-R

[17] Deng, C.Y. and Wang, S.Q. (2012) On Some Characterizations of the Partial Orderings for Bounded Operators. Mathematical Inequalities \& Applications, 15, 619-630. https://doi.org/10.7153/mia-15-54

[18] Liu, F.X. and Yang, H. (2011) Some Results on the Partial Orderings of Block Matrices. Journal of Inequalities and Applications, 2011, 1-7. https://doi.org/10.1186/1029-242x-2011-54

[19] Mitra, S.K. (1987) On Group Inverses and the Sharp Order. Linear Algebra and Its Applications, 92, 17-37. https://doi.org/10.1016/0024-3795(87)90248-5

[20] Baksalary, J.K., Hauke, J. and Styan, G.P.H. (1994) On Some Distributional Properties of Quadratic Forms in Normal Variables and on Some Associated Matrix Partial Orderings. Multivariate Analysis and Its Applications, 24, 111-121.

[21] Baksalary, J.K. and Puntanen, S. (1990) Characterizations of the Best Linear Unbiased Estimator in the General Gauss Markov Model with the Use of Matrix Partial Orderings. $L i$ near Algebra and Its Applications, 127, 363-370. https://doi.org/10.1016/0024-3795(90)90349-H

[22] Puntanen, S. and Styan, G.P.H. (2015) Best Linear Unbiased Estimation in Linear Models (Version 8). StatProb: The Encyclopedia Sponsored by Statistics and Probability Societies.

[23] Stepniak, C. (1987) Ordering of Nonnegative Definite Matrices with Application to Comparison of Linear Models. Linear Algebra and Its Applications, 70, 67-71. https://doi.org/10.1016/0024-3795(85)90043-6

[24] Drazin, M.P. (1978) Natural Structures on Semi Groups with Involution. Bulletin American Mathematical Society, 84, 139-141. https://doi.org/10.1090/S0002-9904-1978-14442-5

[25] Hartwig, R.E. (1980) How to Partially Order Regular Elements? Japanese Journal of Mathematics, 25, 1-13.

[26] Punithavalli, G. (2014) Contributions to the Study on Various Solutions of the Matrix Equation $\mathrm{AXB}=\mathrm{C}$ in Minkowski Space M. PhD Dissertation, Annamalai University, Annamalai Nagar.

[27] Hernnandez, A., Lattanzi, M., Thome, N. and Urquiza, F. (2012) The Star Partial Order and the Eigenprojection at 0 on EP Matrices. Applied Mathematics and Computation, 218, 10669-10678. https://doi.org/10.1016/j.amc.2012.04.034 
Submit or recommend next manuscript to SCIRP and we will provide best service for you:

Accepting pre-submission inquiries through Email, Facebook, LinkedIn, Twitter, etc. A wide selection of journals (inclusive of 9 subjects, more than 200 journals)

Providing 24-hour high-quality service

User-friendly online submission system

Fair and swift peer-review system

Efficient typesetting and proofreading procedure

Display of the result of downloads and visits, as well as the number of cited articles

Maximum dissemination of your research work

Submit your manuscript at: http://papersubmission.scirp.org/

Or contact alamt@scirp.org 